\title{
Very fast inorganic crystal scintillators
}

Ren-Yuan Zhu

Ren-Yuan Zhu, "Very fast inorganic crystal scintillators," Proc. SPIE 10392, Hard X-Ray, Gamma-Ray, and Neutron Detector Physics XIX, 103920G (15 September 2017); doi: 10.1117/12.2274617

SPIE Event: SPIE Optical Engineering + Applications, 2017, San Diego, California, SPIE. United States 


\title{
Very Fast Inorganic Crystal Scintillators \\ Ren-Yuan Zhu*a \\ a 256-48, HEP, California Institute of Technology, Pasadena, CA 91125, USA
}

\begin{abstract}
Future HEP experiments at the energy and intensity frontiers require fast inorganic crystal scintillators with excellent radiation hardness to face the challenges of unprecedented event rate and severe radiation environment. This paper reports recent progress in application of very fast inorganic scintillators in future HEP experiments, such as thin layer of LYSO crystals for a shashlik sampling calorimeter and a precision TOF detector proposed for the CMS upgrade at the HL-LHC, undoped CsI crystals for the Mu2e experiment at Fermilab and yttrium doped $\mathrm{BaF}_{2}$ crystals for the proposed Mu2e-II experiment. Applications for Gigahertz hard X-ray imaging will also be discussed.
\end{abstract}

Keywords: Crystal, scintillator, calorimeter, radiation damage, fast timing

\section{INTRODUCTION}

Fast and radiation hard inorganic crystal scintillators are needed for future HEP experiments at the energy and intensity frontiers. For experiments to be operated at the HL-LHC with $3,000 \mathrm{fb}^{-1}$, for example, crystals should survive an environment with absorbed dose of $100 \mathrm{Mrad}$, charged hadron fluence of $6 \times 10^{14} \mathrm{~cm}^{-2}$ and fast neutron fluence of $3 \times 10^{15}$ $\mathrm{cm}^{-2}$. For future HEP experiments at the intensity frontier, such as Mu2e-II, ultra-fast crystals are needed to face the challenge of unprecedented event rate as well as severe radiation.

Table 1 Inorganic crystals commonly used in HEP experiments

\begin{tabular}{|c|c|c|c|c|c|c|c|c|}
\hline Crystal & $\operatorname{Nal}(\mathrm{TI})$ & CsI(TI) & CsI & $\mathrm{BaF}_{2}$ & BGO & LYSO(Ce) & PWO & $\mathrm{PbF}_{2}$ \\
\hline Density $\left(\mathrm{g} / \mathrm{cm}^{3}\right)$ & 3.67 & 4.51 & 4.51 & 4.89 & 7.13 & & 8.3 & 7.77 \\
\hline Melting Point $\left({ }^{\circ} \mathrm{C}\right)$ & 651 & 621 & 621 & 1280 & 1050 & 2050 & 1123 & 824 \\
\hline Radiation Length (cm) & 2.59 & 1.86 & 1.86 & 2.03 & 1.12 & 1.14 & 0.89 & 0.93 \\
\hline Molière Radius (cm) & 4.13 & 3.57 & 3.57 & 3.10 & 2.23 & 2.07 & 2.00 & 2.21 \\
\hline Interaction Length $(\mathrm{cm})$ & 42.9 & 39.3 & 39.3 & 30.7 & 22.8 & 20.9 & 20.7 & 21.0 \\
\hline Refractive Index a & 1.85 & 1.79 & 1.95 & 1.50 & 2.15 & 1.82 & 2.20 & 1.82 \\
\hline Hygroscopicity & Yes & Slight & Slight & No & No & No & No & No \\
\hline $\begin{array}{l}\text { Luminescence }^{b}(\mathrm{~nm}) \\
\text { (at peak) }\end{array}$ & 410 & 550 & & 220 & 480 & 402 & $\begin{array}{l}425 \\
420\end{array}$ & $?$ \\
\hline Decay Time ${ }^{b}$ (ns) & 245 & 1220 & 26 & $\begin{array}{l}650 \\
0.9\end{array}$ & 300 & 40 & $\begin{array}{l}30 \\
10\end{array}$ & $?$ \\
\hline Light Yield b,c (\%) & 100 & 165 & 4.7 & $\begin{array}{l}36 \\
4.1\end{array}$ & 21 & 85 & $\begin{array}{l}0.3 \\
0.1\end{array}$ & $?$ \\
\hline $\mathrm{d}(\mathrm{LY}) / \mathrm{dT} \mathrm{b}^{\mathrm{b}}\left(\% /{ }^{\circ} \mathrm{C}\right)$ & -0.2 & 0.4 & & -1.9 & -0.9 & -0.2 & -2.5 & $?$ \\
\hline Experiment & $\begin{array}{c}\text { Crystal } \\
\text { Ball }\end{array}$ & $\begin{array}{l}\text { BaBar } \\
\text { BELLE } \\
\text { BES-III }\end{array}$ & $\begin{array}{c}\text { KTeV } \\
\text { S.BELLE } \\
\text { Mu2e-I }\end{array}$ & $\begin{array}{c}\text { (GEM) } \\
\text { TAPS } \\
\text { Mu2e-II? }\end{array}$ & $\begin{array}{c}\text { L3 } \\
\text { BELLE } \\
\text { HHCAL? }\end{array}$ & $\begin{array}{l}\text { COMET \& } \\
\text { CMS (Mu2e } \\
\text { \& SperB) }\end{array}$ & $\begin{array}{c}\text { CMS } \\
\text { ALICE } \\
\text { PANDA }\end{array}$ & $\begin{array}{c}\text { A4 } \\
\text { g-2 } \\
\text { HHCAL? }\end{array}$ \\
\hline
\end{tabular}

a. at peak of emission; b. up/low row: slow/fast component; c. QE of readout device taken out.

*zhu@,hep.caltech.edu; phone 1 (626) 395-6661; fax 1 (626) 395-8728.

Hard X-Ray, Gamma-Ray, and Neutron Detector Physics XIX, edited by Arnold Burger, Ralph B. James,

Michael Fiederle, Larry Franks, Stephen A. Payne, Proc. of SPIE Vol. 10392, 103920G

(C) 2017 SPIE · CCC code: $0277-786 X / 17 / \$ 18 \cdot$ doi: 10.1117/12.2274617 
Table 1 lists basic properties of crystals commonly used to build crystal calorimeters for HEP experiments, where calorimeters with experiment names in brackets were proposed but not constructed. Among the crystals listed in Table 1, bright, fast and radiation hard lutetium yttrium oxyorthosilicate $\left(\mathrm{Lu}_{2(1-x)} \mathrm{Y}_{2 \times} \mathrm{SiO}_{5}\right.$ : $\mathrm{Ce}$ or LYSO) crystals were proposed for a LYSO/W/Quartz capillary sampling calorimeter [1] and a precision time of flight (TOF) layer for the CMS upgrade for the HL-LHC [2]. LYSO crystals are also proposed to construct a total absorption calorimeter for the COMET experiment at KEK [3], and a 3D calorimeter for the proposed HERD experiment in space [4]. Undoped CsI crystals are used to construct a calorimeter for the Mu2e experiment at Fermilab [5]. $\mathrm{BaF}_{2}$ crystals have a unique fast scintillation light with sub-ns decay time, but also a slow scintillation light component with $600 \mathrm{~ns}$ decay time. Recently developed yttrium doped $\mathrm{BaF}_{2}$ crystals with effective slow component suppression have a great potential for an extra fast calorimeter for Mu2e-II [6]. They may also be used in the proposed MaRIE facility at Los Alamos [7], where unprecedented fast X-ray imaging requires ultra-fast detector response for hard X-rays [8].

\section{PULSE SHAPE AND RISE TIME OF FAST SCINTILLATORS}

Crystal time resolution depends on the signal to noise ratio for the rise time measurement. While the intrinsic rising time of most crystals is as fast as a few tens ps [9], Fig. 1 shows the rise time measured for ten crystal samples of $1.5 \mathrm{X}_{0}$ size by using a Hamamatsu R2059 PMT and a fast digital scope. The fast rise time of about 1.5 and $1.6 \mathrm{~ns}$ observed respectively for $\mathrm{BaF}_{2}$ and LYSO is limited by the PMT rise time $1.3 \mathrm{~ns}$ at 2,500 V bias and the rise time of $0.14 \mathrm{~ns}$ of the Agilent MSO9254A (2.5 $\mathrm{GHz}$ ) DSO. The measured rise time is also faster for the same crystal with a black wrapping, where the light propagation in crystal is minimized [10].
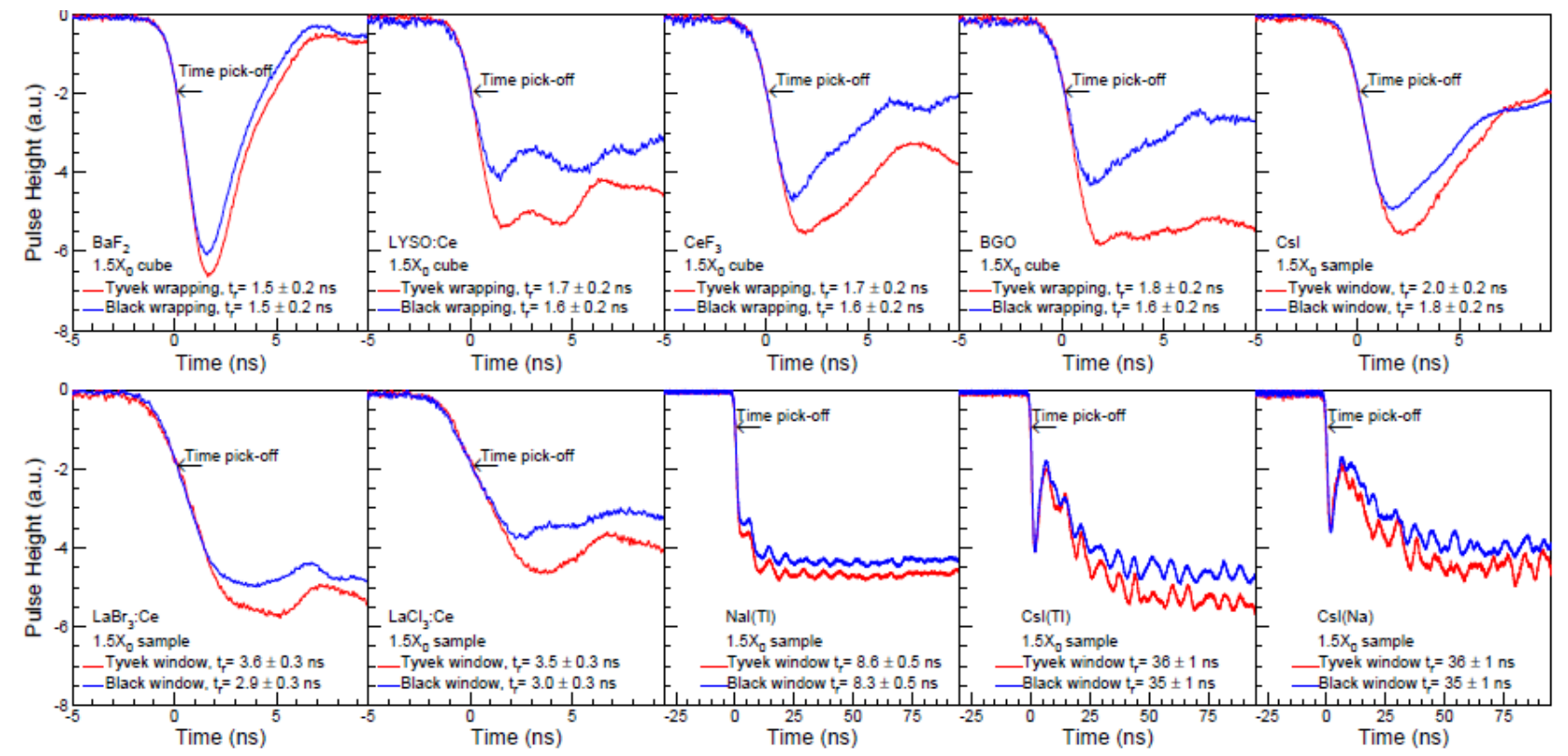

Fig. 1. Scintillation rising time measured for ten crystal samples of $1.5 \mathrm{X}_{0}$

Table 2 lists the figure of merit values on time resolution for various crystal detectors, which is defined as the light output in the $1^{\text {st }}$, or the $1^{\text {st }} 0.1$, ns [10]. It is clear that the best crystal scintillators for ultra-fast timing are $\mathrm{BaF}_{2}$, LSO:Ca,Ce and LYSO:Ce. $\mathrm{LaBr}_{3}$ is a material with high potential theoretically, but suffers from scattering centers in the crystal as well as its intrinsic hygroscopicity. On the other hand, the decay time of most fast scintillators at a level of few tens ns would cause pile-up for calorimeters designed for very fast rate capability and $\mathrm{GHz}$ x-ray imaging with a x-ray frame rate of a few ns. It thus is crucial to develop very fast inorganic scintillator capable to provide clean signal pulse of a few ns width. Among all scintillators shown in Fig. 1, the $\mathrm{BaF}_{2}$ crystals are the only one with this capability provided that its slow scintillation component is suppressed. 
Table 2. Figure of Merit for Time Resolution for Various Crystal Scintillators

\begin{tabular}{|c|c|c|c|c|c|c|c|c|c|}
\hline $\begin{array}{c}\text { Crystal } \\
\text { Scintillators }\end{array}$ & $\begin{array}{c}\text { Relative LY } \\
(\%)\end{array}$ & $\mathrm{A}_{1}(\%)$ & $\tau_{1}(\mathrm{~ns})$ & $\mathrm{A}_{2}(\%)$ & $\tau_{2}$ (ns) & $\begin{array}{c}\text { Total LO } \\
(\text { p.e./MeV, } \\
\text { XP2254B) }\end{array}$ & $\begin{array}{c}\text { LO in 1ns } \\
\text { (p.e./MeV, } \\
\text { XP2254B) }\end{array}$ & $\begin{array}{c}\text { LO in 0.1ns } \\
\text { (p.e./MeV, } \\
\text { XP2254B) }\end{array}$ & $\begin{array}{c}\text { LY in 0.1ns } \\
\text { (photons/MeV) }\end{array}$ \\
\hline \hline $\mathrm{BaF}_{2}$ & 40.1 & 91 & 650 & 9 & 0.9 & 1149 & 71.0 & 11.0 & 136.6 \\
\hline $\mathrm{LSO}: \mathrm{Ca}, \mathrm{Ce}$ & 94 & 100 & 30 & & & 2400 & 78.7 & 8.0 & 110.9 \\
\hline $\mathrm{LSO} / \mathrm{LYSO}_{\mathrm{Cl}}$ & 85 & 100 & 40 & & & 2180 & 53.8 & 5.4 & 75.3 \\
\hline $\mathrm{CeF}_{3}$ & 7.3 & 100 & 30 & & & 208 & 6.8 & 0.7 & 8.6 \\
\hline $\mathrm{BGO}$ & 21 & 100 & 300 & & & 350 & 1.2 & 0.1 & 2.5 \\
\hline $\mathrm{PWO}$ & 0.377 & 80 & 30 & 20 & 10 & 9.2 & 0.42 & 0.04 & 0.4 \\
\hline $\mathrm{LaBr}_{3}: \mathrm{Ce}$ & 130 & 100 & 20 & & & 3810 & 185.8 & 19.0 & 229.9 \\
\hline $\mathrm{LaCl}: \mathrm{Ce}$ & 55 & 24 & 570 & 76 & 24 & 1570 & 49.36 & 5.03 & 62.5 \\
\hline $\mathrm{Nal:} \mathrm{Tl}$ & 100 & 100 & 245 & & & 2604 & 10.6 & 1.1 & 14.5 \\
\hline $\mathrm{Csl}$ & 4.7 & 77 & 30 & 23 & 6 & 131 & 7.9 & 0.8 & 10.6 \\
\hline $\mathrm{Csl}: \mathrm{Tl}$ & 165 & 100 & 1220 & & & 2093 & 1.7 & 0.2 & 4.8 \\
\hline $\mathrm{Csl}: \mathrm{Na}$ & 88 & 100 & 690 & & & 2274 & 3.3 & 0.3 & 4.5 \\
\hline
\end{tabular}

\section{QUALITY OF PREPRODUCTION CSI CRYSTALS FOR MU2E}

The Mu2e experiment at Fermilab is constructing a fast calorimeter using undoped CsI crystals. 72 preproduction CsI crystals from three vendors were characterized. Fig. 2 compares measured data with the Mu2e specifications (red dashed lines), showing that most crystals meet the specifications on light output, FWHM energy resolution, light response uniformity, F/T (Fast/Total) ratio, $\gamma$-ray induced noise and radiation hardness. Some crystals failed specifications on F/T ratio and $\gamma$-ray induced noise because of significant slow scintillation component. Excellent correlations have been observed between the light output and the energy resolution, and the dark current, the $\gamma$-ray induced readout noise and the $\mathrm{F} / \mathrm{T}$ ratio, confirming that they are of the same origin [11].


Fig. 2. Quality of preproduction undoped CsI crystals of $3.4 \times 3.4 \times 20 \mathrm{~cm}$ 


\section{YTTRIUM DOPED BAF 2 CRYSTALS}

$\mathrm{BaF}_{2}$ crystal has a very fast scintillation component peaked at $220 \mathrm{~nm}$ with sub-ns decay time, which provides a solid foundation for a very fast calorimetry. It, however, has also a slow scintillation component peaked at $300 \mathrm{~nm}$ with $600 \mathrm{~ns}$ decay time and a five times brightness of its fast component, which would cause pileup in an environment of fast rate. Two approaches are used to reduce the pileup caused by slow component: selective doping with rare earth (La, Y, and $\mathrm{Ce})$ in $\mathrm{BaF}_{2}$ [12] and selective readout with solar blind photodetector [13]. The top left photo of Fig. 3 shows a set of nine yttrium doped $\mathrm{BaF}_{2}$ crystal samples of $\Phi 18 \times 21 \mathrm{~mm}$ doped with yttrium from 0 to $7 \mathrm{~mol} \%$ from Beijing Glass Research Institute (BGRI). The top middle and right plot shows their x-ray excited luminescence (XEL) and transmittance spectra respectively. The bottom left plot shows their light output as a function of integration time. The bottom middle and right plots show their light output in 50 and 2,500 ns gates and the corresponding ratios respectively as a function of the level of yttrium doping in mol\%. It was found that the optimized yttrium doping in $\mathrm{BaF}_{2}$ is about 5 mol\%, which increases the F/S (Fast/Slow) ratio from $1 / 5$ to $5 / 1$ while the amount of fast light is unchanged. The crystals of this nature is expected to find a broad application in future HEP experiments and GHz x-ray imaging.
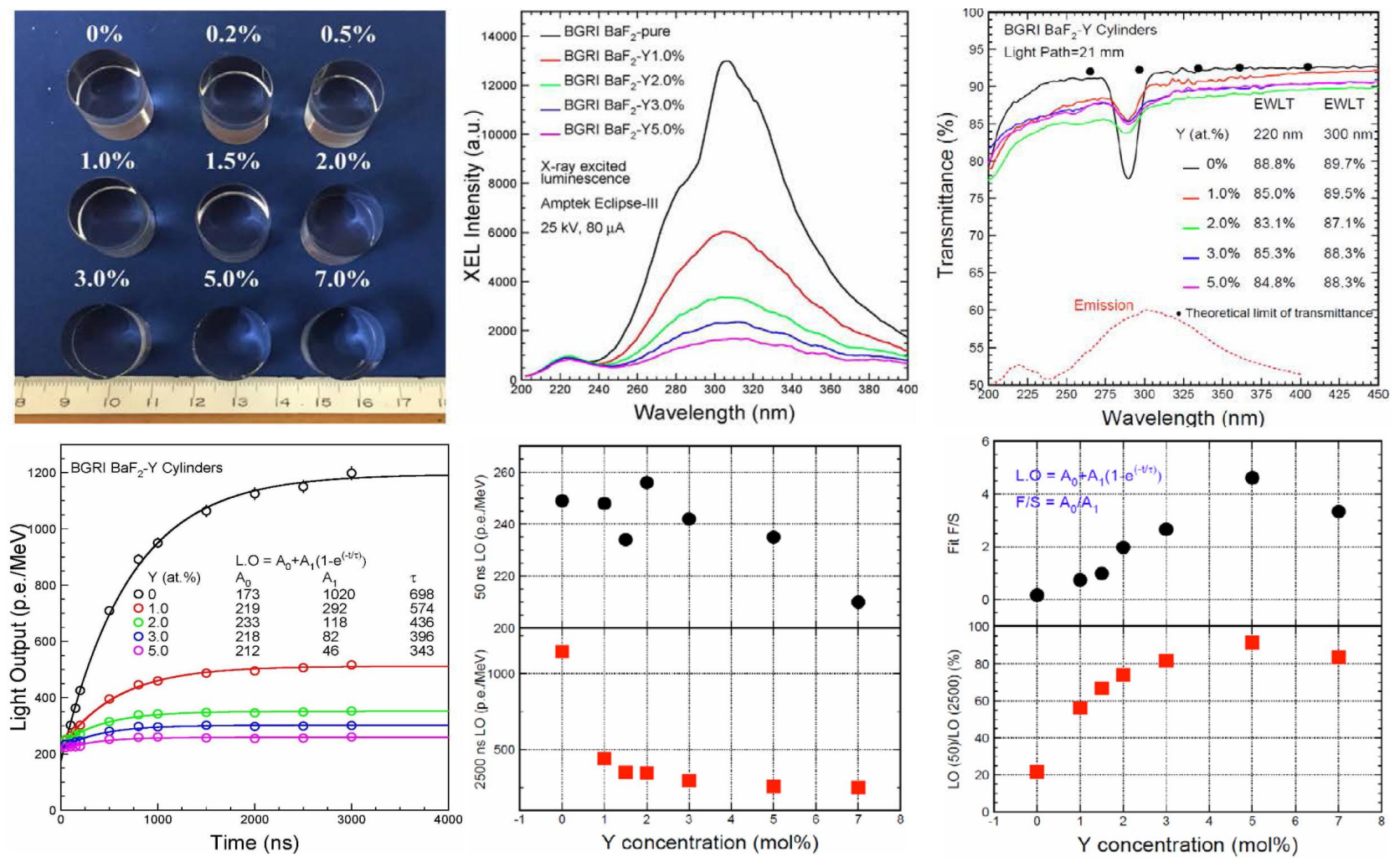

Fig. 3. Performance of Yttrium doped $\mathrm{BaF}_{2}$ Crystals of $\Phi 18 \times 21 \mathrm{~mm}$

\section{NEUTRON INDUCED RADIATION DAMAGE IN FAST CRYSTALS}

One crucial issue for applications of scintillation crystals in future HEP experiments is radiation damage in severe radiation environment, such as the HL-LHC. While radiation damage induced by ionization dose in inorganic crystal scintillators is well understood, investigations are on-going to understand radiation damage caused by hadrons, including both charged hadrons and neutrons. Two neutron irradiation experiments were carried out in 2015 (Exp. 6991) and 2016 (Exp. 7332) in the East Port of the Target-4 area (top left plot in Fig. 4), about $1.2 \mathrm{~m}$ away from the neutron production target at the Weapons Neutron Research facility of the Los Alamos Neutron Science Center (WNR of LANSCE). The top middle plot of Fig. 4 shows the particle rate as a function of energy for neutrons, photons and protons, tallied on the 
largest sample volume (averaging) around the samples, which are calculated by using the Monte Carlo N-Particle eXtended (MCNPX) transport package developed by LANL. In the 2016 run, 36 samples of $5 \mathrm{~mm}$ thick were irradiated for 21.2, 46.3 and 120 days in three groups of 12 each (top right photo of Fig. 4), where half samples in each group were shielded by $5 \mathrm{~mm} \mathrm{~Pb}$, and other half not. The corresponding fast neutron $(>1 \mathrm{MeV})$ fluence and ionization dose were 3.94, 8.58 and $19.3 \times 10^{14} \mathrm{n} / \mathrm{cm}^{2}$ and 42/30, 52/65 and 207/147 Mrad respectively for three groups without/with lead shielding.
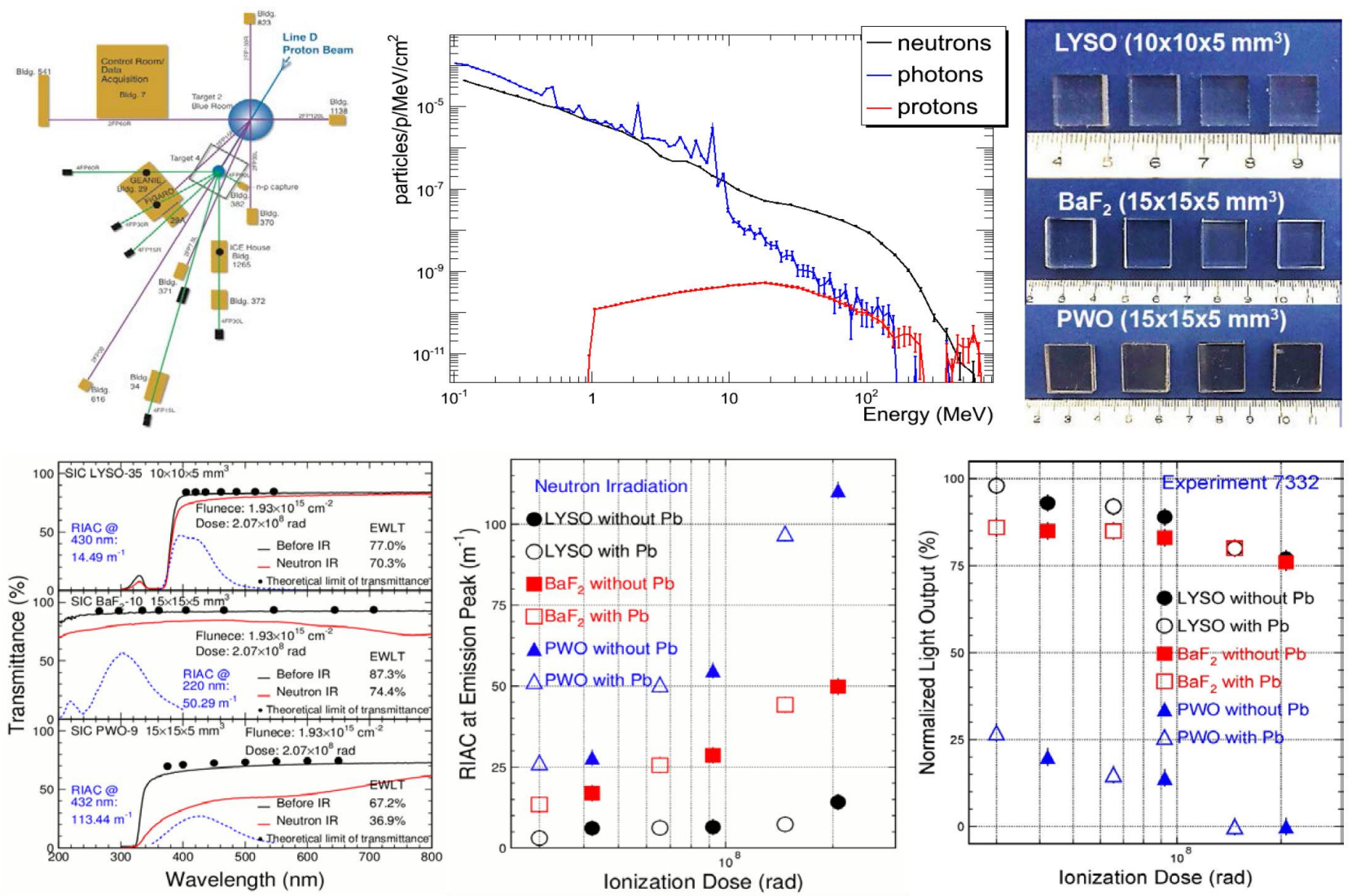

Fig. 4. Neutron Irradiation Experiment for LYSO, BaF2 and PWO crystals at LANSCE

The bottom left plot of Fig. 4 shows transmittance spectra before and after irradiation for one sample each of LYSO (top), $\mathrm{BaF}_{2}$ (middle) and PWO (bottom) without lead shielding in the group 3 of the 2016 run. Also listed in the figure is the numerical values of the emission weighted longitudinal transmittance (EWLT) and the radiation induced absorption coefficient (RIAC) after the irradiation. It is clear that LYSO and $\mathrm{BaF}_{2}$ are much more radiation hard than PWO. The bottom middle and right plots of Fig. 4 present the RIAC and the normalized light output (LO) as a function of the ionization dose, showing that the contribution of fast neutron induced damage, if any, is much less than that from the ionization dose. This observation confirms an early observation of no neutron specific damage in PWO crystals [14], and indicates that neutrons are very different from charged hadrons in hadron induced radiation damage in scintillating crystals.

\section{SUMMARY}

LYSO is a robust scintillators against ionization dose as well as charged and neutral hadrons expected at the HL-LHC. Commercially available undoped CsI crystals satisfy the Mu2e requirements. Commercially available undoped $\mathrm{BaF}_{2}$ crystals provide sufficient fast light with sub-ns decay time and excellent radiation hardness beyond 100 Mrad and 
$1 \times 10^{15} \mathrm{p} \mathrm{cm}^{-2}$. They promise a very fast and robust calorimeter in a severe radiation environment. Yttrium doping in $\mathrm{BaF}_{2}$ crystals increases the $\mathrm{F} / \mathrm{S}$ ratio from $1 / 5$ to $5 / 1$ without using a selected readout, while maintaining the amount of fast scintillation light unchanged. The slow contamination at this level is already much less than the commercially available undoped CsI crystals. This novel crystal promises a very fast calorimetry for future HEP experiments and an effective frontend detector for $\mathrm{GHz} x$-ray imaging. Results of the experiments 6991 and 7332 at LANSCE show fast neutrons up to $2 \times 10^{15} \mathrm{n} / \mathrm{cm}^{2}$ do not damage LYSO, $\mathrm{BaF}_{2}$ and PWO crystals. Our plan is to further investigate radiation hardness of $\mathrm{Y}: \mathrm{BaF}_{2}$ crystals, and pay special attention to photodetector with DUV response: LAPPD, Si or diamond based solid state detector [15].

\section{ACKNOWLEDGEMENTS}

This work is supported by the U.S. Department of Energy, Office of High Energy Physics program under Award Number DE-SC0011925.

\section{REFERENCES}

[1] L. Y. Zhang, R. H. Mao, F. Yang, et al., "LSO/LYSO Crystals for Calorimeters in Future HEP Experiments," IEEE Trans. Nucl. Sci, vol. 61, pp. 483-488, 2014.

[2] T. Tabarelli de Fatis., "Precision timing studies and detector concept proposal," presentation given in the CMS general meeting on November 16, 2016.

[3] K. Oishi, "An LYSO Electromagnetic Calorimeter for COMET at J-Park," paper O47-4 presented in IEEE NSS2014, Seattle, USA.

[4] S.N. Zhang, O. Adriani, S. Albergo, et al., "The High Energy cosmic-Radiation Detection (HERD) Facility onboard China's Future Space Station," Proc. SPIE Int. Soc. Opt. Eng., vol. 9144, pp. 91440X, 2014.

[5] G. Pezzullo, "Design, status and perspectives for the Mu2e crystal calorimeter", in these proceedings.

[6] The Mu2e collaboration, mu2e-doc-db-10655 (http://mu2e-docdb.fnal.gov:8080/cgibin/ShowDocument?docid=10655).

[7] http://www.lanl.gov/science-innovation/science-facilities/marie/index.php.

[8] Z. Wang, C. W. Barnes, J. S. Kapustinsky, et al., "Thin scintillators for ultrafast hard X-ray imaging," Proc. SPIE 9504, Photon Counting Applications, Paper 95040N, May 6, 2015.

[9] Derenzo S E, Weber M J, Moses W W, and Dujardin C, 2000, IEEE Trans. Nucl. Sci., 47, 860.

[10] R.-Y. Zhu,http://www.hep.caltech.edu/ zhu/talks/ryz_110428_time_resolution.pdf

[11] F. Yang, L.Y. Zhang and R.-Y. Zhu, "Slow Scintillation Component and Radiation Induced Readout Noise in Pure CsI Crystals", Paper N07-9 in 2016 IEEE NSS conference record.

[12] B.P. Sobolev et al., "Suppression of BaF2 Slow Component of $x$-ray Luminescence in Non-Stoichiometric Ba0.9R0.1F2 Crystals (R=Rare Earth Element)", Proceedings of the Material Research Society: Scintillator and Phosphor Materials, 00.227-283, 1994..

[13] F. Yang, J.F. Chen, L.Y. Zhang and R.-Y. Zhu, "Development of BaF2 Crystals for Future HEP Experiments at the Intensity Frontiers", Paper N36-7 in 2016 IEEE NSS conference record..

[14] R. Chipaux et al., in Proc. 8th Int. Conference on Inorganic Scintillators, SCINT2005, 369, 2005.

[15] E. Monroy, F. Omnes and F. Calle, "Wide-bandgap semiconductor ultraviolet photodetectors," IOP science 2003 Semicond. Sci. Technol. 18 R33. 